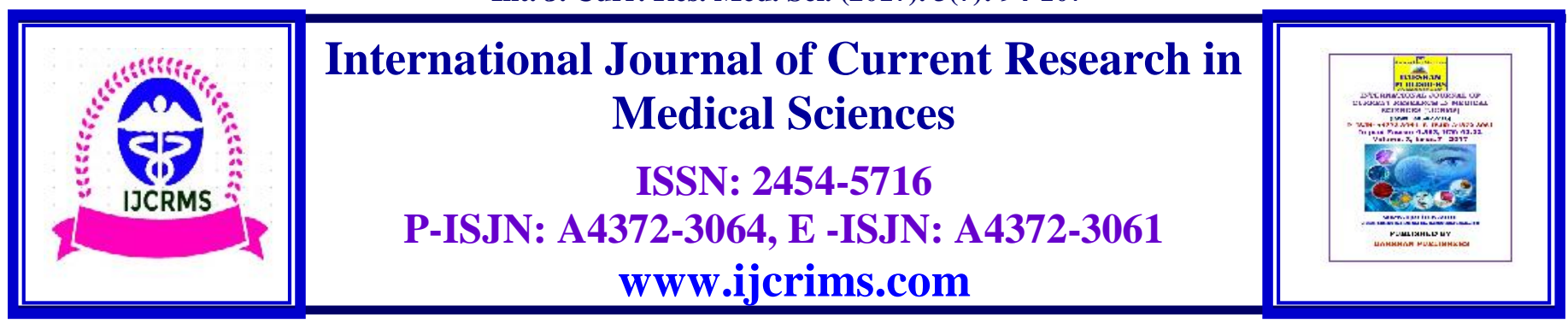

\title{
Association and impact of non-alcoholic fatty liver disease on thyroid function
}

\author{
Arafat Kassem¹, Farag Khalil', Mokhtar Ragab Ramadan², Mohamed Rashed ${ }^{3}$ \\ ${ }^{1}$ Department of Internal Medicine, Faculty of Medicine, Al-Azhar University ,Cairo, Egypt \\ ${ }^{2}$ Department of Radiology, Faculty of Medicine, Al-Azhar University ,Cairo, Egypt \\ ${ }^{3}$ Department of Clinical Pathology, Faculty of Medicine, Al-Azhar University ,Cairo, Egypt
}

\begin{abstract}
Nonalcoholic fatty liver disease (NAFLD) means accumulation of fat mainly triglyceride exceeding $5 \%$ of liver weight, affecting approximately $20 \%$ of population in developed countries. Thyroid dysfunction have been frequently associated with non-alcoholic fatty liver disease. The aim of this study is to determine any association between nonalcoholic fatty liver disease and thyroid dysfunction and to evaluate the impact of non-alcoholic fatty liver disease on thyroid function tests. Subjects and methods: This study was carried out on sixty adult men and women with nonalcoholic fatty liver disease and sixty age- and sex-matched males and females without the disease. NAFLD patients were subjected to a full assessment of medical history, physical examination, abdominal ultrasonography as well as routine laboratory tests. Patients and controls underwent liver function and thyroid function testing including thyroid antibodies. Results: Thirteen patients with NAFLD (21.7\%) were found to have thyroid dysfunction. The most common thyroid dysfunction was subclinical hypothyroidism which was found in $10(16.7 \%)$ NAFLD patients followed by overt hypothyroidism in 3 patients $(5 \%)$. There were statistically significant difference between patients and controls as regard to TSH ( $\mathrm{p}=0.0001)$, FT4 ( $\mathrm{p}=0.005)$, FT3 ( $\mathrm{P}=0.043)$, anti-TPO ( $\mathrm{p}=0.047)$, anti-TG ( $\mathrm{p}=0.001)$, IR $(\mathrm{p}=0.024)$, insulin $(\mathrm{p}=0.002)$, and Leptin $(\mathrm{p}=0.002)$. TSH concentration was significantly correlated with ALT ( $\mathrm{r}=$ 0.381), GGT ( $\mathrm{r}=0.354)$, HbA1C ( $\mathrm{r}=0.288)$, triglyceride ( $\mathrm{r}=0.368)$, FT4 ( $\mathrm{r}=0.414)$, FT3( $\mathrm{r}=0.231)$, anti-TPO( $\mathrm{r}=0.298)$, serum insulin ( $r=0.287)$, insulin resistance $(I R)(r=0.453)$, and serum Leptin $(r=0.360)$ levels. In our patients, hepatic steatosis in the form of increased liver echogenecity was present in almost all patients (100\%), and hepatomegaly in twenty-four patients (40\%). Thus. Hepatic steatosis were correlated significantly with ALT, GGT, HbA1C, triglyceride, HDL-C, TSH, FT4, FT3, anti-TPO, anti-TG, serum insulin, insulin resistance (IR), and serum Leptin. Conclusion: Thyroid dysfunctions are common in NAFLD patients, with subclinical hypothyroidism hypothyroidism being the most prevalent one. Hepatic steatosis has shown an evident positive correlation with elevated TSH as well as thyroid autoantibodies.
\end{abstract}

Keywords: Non-alcoholic fatty liver disease, Hypothyroidism, Insulin resistance, Hyperlipidemia, Leptin. 


\section{Introduction}

Nonalcoholic fatty liver disease (NAFLD) is characterized by excessive hepatic accumulation of triglycerides and free fatty acids in the liver (1). The incidence of NAFLD is increasing rapidly, and it is the most common cause of abnormal liver function results worldwide (2). The increase in the prevalence of NAFLD has been attributed to the global increase in the prevalence of obesity and other metabolic risk factors. Advanced age and metabolic disorders such as type 2 diabetes mellitus, impaired glucose tolerance, and central obesity, are among the risk factors for NAFLD (3). Cryptogenic cirrhosis is a term used for patients with liver cirrhosis who lack any identifiable viral, alcoholic, autoimmune or drug-related causes for the condition. Many clinicians now believe that a considerable number of these patients have cirrhosis due to nonalcoholic steatosis hepatitis (NASH) (4). NAFLD has broad pathological spectrum, ranging from simple steatosis to NASH, and potentially progressing to fibrosis and cirrhosis (5). After fat infiltrates the liver, progression to hepatocellular inflammation and fibrosis may occur.

The thyroid gland is significantly involved in energy homeostasis, lipid and carbohydrate metabolism, regulation of body weight and adipogenesis. Subclinical and overt hypothyroidism has been associated with metabolic syndrome, cardiovascular mortality and disturbance in lipid metabolism (6). Hypothyroidism is an endocrine as well as autoimmune disorder. It is caused by decrease in thyroid function. The patient of hypothyroidism is obese. There may have diabetes, hypercholesterolemia, and may present with weight gain, puffy face, hoarse and croaky voice, non-pitting edema in legs, loss of outer third of eyebrow, dry skin and bradycardia (7). There is also delayed relaxation of ankle jerk and hypertension. Laboratory finding of hypothyroidism include low FT3 and FT4 but raised serum level of TSH. Since hypothyroidism is an autoimmune disease, it may be associated with other autoimmune disease like pernicious anemia and diabetes. In this study we try to find out any association between non-alcoholic fatty liver disease and thyroid abnormalities and to evaluate the impact of non-alcoholic fatty liver disease on thyroid function tests in Egyptian patients.

\section{Materials and Methods}

This case-control study consisted of sixty adult men and women with non-alcoholic fatty liver disease in the period between May 2015 to April 2016 and sixty age- and sex-matched males and females without the disease. The diagnosis of NAFLD was based on the results of abdominal ultrasonography, after excluding heavy alcohol consumption, and viral, or other liver diseases. The subjects with NAFLD were drawn from Outpatients Clinic of the Internal Medicine Department of El-Husein University Hospital and the controls were members of staff of the hospital. The Ethical Research and Review Committee of the Hospital approved the study protocol, and informed consent was obtained from the participants.

The inclusion criteria included NAFLD of either sex age 18 years or more. However, the following subjects were excluded: (a) those with renal disease; type 1 or 2 diabetes; glucocorticoid therapy, overt hypothyroidism, and Cushing's disease); (b) those with known thyroid disease, current or past history of thyroid hormone or antithyroid drug intake, thyroid alterations in volume and morphology at ultrasound, (c) those with any laboratory or clinical evidence suggesting an alternate or coexistent underlying chronic liver disease including hepatic virus infections (Hepatitis A-E), autoimmune hepatitis, metabolic hepatic disease, and (d) those with history of alcohol consumption).

All patients were subjected to full medical history and were clinically examined for establishment of fatty liver. Ultrasonography of hepatobiliary system of all patients was done. Blood was examined for total bilirubin, alanine aminotransferase (ALT), aspartate aminotransferase (AST), gamma-glutamyl transferase (GGT), and prothrombin time (PT). Serum triglycerides (TG), Total Cholesterol (TC) and high density lipoprotein (HDL cholesterol) 
were measured photo metrically using commercial kit provided from Spinreact, and LDL was calculated by the equation ( $\mathrm{LDL}=$ cholesterol - triglycerides/5 -HDL). Fasting blood glucose was measured according to glucose-oxidase method. Glycated hamoglobin A1C was measured using a column chromatography method by commercial kit provided from Biosystem Company. After final elution the result was determined photometrically using Biosystem photometer (Reference range 5.1-6.4\%). \%). Insulin level: was estimated according to Angel (1988) (8) using a commercially available ELISA kit which was modified for use in microtiter plates. The adapted assay, is based on the binding of porcine anti-guinea pig insulin antibodies to microtiter plates and uses insulinperoxidase conjugate as displacer. IR was calculated using the homeostasis model assessment for IR formula: fasting glucose $(\mathrm{mg} / \mathrm{dL}) \quad X$ fasting insulin $(\mu \mathrm{U} / \mathrm{mL}) / 405$. Serum leptin level: was measured by a commercially available ELISA kit (The DSL- 10-23100 Human Leptin ELISA Kit; Diagnostic System laboratories, Webster, Texas). This assay is a direct Sandwich ELISA based, sequentially, on capture of human leptin molecules from samples to the wells of a microtiter plate coated by pre-titered amount of polyclonal rabbit anti-human leptin antibodies, and wash away of unbound materials from samples, then, binding of a biotinylated monoclonal antibody to the captured human leptin, and conjugation of alkaline phosphatase to biotinyiated antibodies, after that, wash away of free antibody-enzyme conjugates. Assessment of thyroid function was performed using the following: Thyroid stimulating hormone (TSH): was measured using immunometric assays ( IMMULITE 2000 Third Generation; Diagnostic ProductsCorporation, Los Angles, California, USA), Refrence value was 0.27-4.2 IU/ml. Free serum tri-iodothyronin (FT3) level was determined using IMMULITE 2000FT3, competitive, analog-based immunoassay for quantitative estimation of FT3 in serum on an IMMULITE 2000 system, Refrence value was $2.57-4.43 \mathrm{pg} / \mathrm{ml}$. Free serum thyroxin level (FT4) was measures IMMULITE 2000 FT4, solid phase chemiluminescent competitive immunoassay method for the quantitative determination of FT4 in serum on an IMMULITE 2000 system, Refrence value was 0.93-1.71 ng/ml. Antithyreoglobulin (anti-TG) antibodies and antithyroid peroxidase (anti-TPO) were assayes using an Immunoradiometric Assay. The refrence values were less than $20 \mathrm{IU} / \mathrm{ml}$ for anti-TG antibodies and less than $35 \mathrm{IU} / \mathrm{ml}$ for anti-TPO (7).

\section{Results}

The mean age of patients was $35.3 \pm 12.4$ years. Baseline clinical data of patients are shown in table 1.

Table 1: Demographic data for patients and controls:

\begin{tabular}{llll}
\hline Demographic data & Patients & Controls & P value \\
Age (years) & $\mathbf{3 5 . 3} \pm \mathbf{1 2 . 4 1}$ & $\mathbf{3 7 . 0} \pm \mathbf{1 3 . 1 4}$ & $\mathbf{0 . 4 2 6}$ \\
$\begin{array}{l}\text { Mean } \pm \text { SD } \\
\text { Sex }[\text { No. }(\%)]\end{array}$ & & & \\
Male & $\mathbf{2 0}(\mathbf{3 3 . 3 \%})$ & $\mathbf{2 0}(\mathbf{3 3 . 3 \%})$ & $\mathbf{0 . 0 6 4}$ \\
Female & $\mathbf{4 0}(\mathbf{6 6 . 7 \%})$ & $\mathbf{4 0}(\mathbf{6 6 . 7 \% )}$ & \\
\hline
\end{tabular}

This table shows no statistically significant difference between patients and controls as regard age and sex.

There were 40 females $(66.7 \%)$ and 20 males $(33.3 \%)$.

Ultrasonography of hepatobiliary system to assess the liver echotexture and hepatic steatosis was done and revealed that twenty-four patients (40\%) had hepatomegaly and $100 \%$ of patients had both increased liver echogenecity and hepatomegaly as shown in table 2. 
Int. J. Curr. Res. Med. Sci. (2017). 3(7): 94-107

Table 2: Ultrasonography of hepatobiliary system

\begin{tabular}{lll}
\hline & No. of patients & Percent \\
Increased liver echogenicity & 60 & $100 \%$ \\
Hepatomegally & 24 & $40 \%$ \\
\hline
\end{tabular}

Baseline laboratory parameters of our patients and controls are shown in table 3 . There were statistically significant difference between patients and controls as regard to alanine aminotransferase (ALT), gamma-glutamyl transferase (GGT), fasting blood sugar (FBS), glycated hemoglobin (HbA1C), total cholesterol, low-density lipoprotein cholesterol (LDL-C), high-density lipoprotein cholesterol (HDL-C), triglycerides, thyroid stimulating hormone (TSH), free thyroxine (FT4), free tri-idothyronine (FT3), anti-thyroid peroxidase (anti-TPO), antithyroglobulin (anti-TG),serum insulin, insulin resistance (IR), and serum leptin, but there were no statistically significant difference between our patients and controls as regard to aspartate aminotransferase (AST), total bilirubin, and prothrombin time as shown in table 3 .

Table 3: Laboratory parameters of patients and controls:

\begin{tabular}{|c|c|c|c|}
\hline \multirow[t]{2}{*}{ Parameter } & \multicolumn{2}{|l|}{ Range \pm SD } & \multirow[t]{2}{*}{$P$ value } \\
\hline & Patients & Controls & \\
\hline ALT (IU/L) & $61.68 \pm 7.38$ & $25.7 \pm 5.46$ & $0.004 *$ \\
\hline AST( IU/L) & $49.73 \pm 7.57$ & $30.17 \pm 2.07$ & 1.186 \\
\hline GGT (IU/L) & $94.53 \pm 19.97$ & $64.1 \pm 9.9$ & $0.001 *$ \\
\hline Total bilirubin (mg/dl) & $0.94 \pm 0.18$ & $0.69 \pm 0.13$ & 1.381 \\
\hline Prothrombin time (seconds) & $13.67 \pm 1.41$ & $11.57 \pm 0.5$ & 0.542 \\
\hline FBS $(\mathrm{mg} / \mathrm{dl})$ & $109.57 \pm 7.52$ & $94.47 \pm 5.63$ & $0.037 *$ \\
\hline $\operatorname{HbA1C}(\%)$ & $6.16 \pm 0.24$ & $4.48 \pm 0.46$ & $0.034 *$ \\
\hline Total cholesterol (mg/dl) & $221.17 \pm 15.7$ & $168.07 \pm 7.6$ & $0.009 *$ \\
\hline LDL-C (mg/dl) & $150.17 \pm 15.4$ & $73.47 \pm 2.74$ & $0.003 *$ \\
\hline HDL-C (mg/dl) & $31.47 \pm 3.6$ & $62.3 \pm 7.47$ & $0.006^{*}$ \\
\hline Triglycerides(mg/dl) & $196.19 \pm 40.43$ & $139.43 \pm 5.94$ & $0.0001 *$ \\
\hline TSH (IU/ml) & $4.45 \pm 1.39$ & $2.31 \pm 0.81$ & $0.0001 *$ \\
\hline FT4 (ng/ml) & $0.93 \pm 0.18$ & $1.48 \pm 0.2$ & $0.005 *$ \\
\hline FT3 $(\mathrm{pg} / \mathrm{ml})$ & $3.12 \pm 0.89$ & $3.34 \pm 0.32$ & $0.043 *$ \\
\hline Anti-TPO (IU/ml) & $33.43 \pm 7.1$ & $21.3 \pm 2.35$ & $0.047 *$ \\
\hline Anti-TG (IU/ml) & $26.37 \pm 7.8$ & $17.6 \pm 1.3$ & $0.001 *$ \\
\hline Leptin $(\mathrm{ng} / \mathrm{ml})$ & $21.8 \pm 3.69$ & $10.4 \pm 1.8$ & $0.002 *$ \\
\hline Insulin(mIU/ml) & $13.4 \pm 2.1$ & $7.1 \pm 2.08$ & $0.002 *$ \\
\hline IR & $5.59 \pm 1.16$ & $3.25 \pm 1.21$ & $0.024 *$ \\
\hline
\end{tabular}

*Statistically significant, ALT $=$ Alanine aminotransferase, AST $=$ Aspartate aminotransferase, GGT $=$ Gmma glutamyl transferase, FBS= Fasting blood suger, HbA1c=Haemoglobin A1c, LDL-C= Low dendity lipoprotein, HDL-C= High density lipoprotein, TSH= Thyroid stimulating hormone, FT4= Free thyroxine, FT3= Free tri-idothyronine, Anti-TPO= Anti-thyroid peroxidase, Anti-TG= Anti-thyroglobulin .

Laboratory thyroid abnormalities were present in 13 patients $(21.7 \%)$, from whom 10 patients (16.7\%) had subclinical hypothyroidism and the other three patients (5\%) had overt hypothyroidism as shown in table 4. 
Int. J. Curr. Res. Med. Sci. (2017). 3(7): 94-107

Table 4: Pattern of thyroid profile in fatty liver patients

\begin{tabular}{lll}
\hline TSH & Frequency & Percent \\
Subclinical & 10 & 16.7 \\
hypothyroidism & & \\
Euthyroid state & 47 & 78.3 \\
Hypothyroidism & 3 & 5 \\
\hline
\end{tabular}

There were statistically significant correlation between serum thyroid stimulating hormone (TSH) in our patients with ALT, GGT, HbA1C, triglyceride, FT4, FT3, anti-TPO, serum insulin, insulin resistance (IR), and serum Leptin levels but there were no statistically significant correlation between serum TSH with AST, total bilirubin, prothrombin time, FBS, total cholesterol, LDL-C, HDL-C, and anti-TG as shown in table 5 .

Table 5: Correlation between serum TSH With laboratory parameters of our patients:

\begin{tabular}{llll}
\hline Variables & TSH & P value & \\
& $\mathbf{r}$ & $\mathbf{0 . 0 0 2}$ & HS \\
ALT & $\mathbf{0 . 3 8 1}$ & $\mathbf{1 . 2 9 7}$ & NS \\
AST & $\mathbf{0 . 5 9 9}$ & $\mathbf{0 . 0 0 5}$ & HS \\
GGT & $\mathbf{0 . 3 5 4}$ & $\mathbf{1 . 3 9 0}$ & NS \\
Total bilirubin & $\mathbf{0 . 5 7 7}$ & $\mathbf{2 . 5 3 1}$ & NS \\
Prothrombin time & $\mathbf{0 . 7 7 0}$ & $\mathbf{2 . 2 1 5}$ & NS \\
FBS & $\mathbf{0 . 6 3 7}$ & $\mathbf{0 . 0 4}$ & S \\
HbA1C & $\mathbf{0 . 2 8 8}$ & $\mathbf{0 . 0 0 3}$ & HS \\
Triglyceride & $\mathbf{0 . 3 6 8}$ & $\mathbf{1 . 6 6 1}$ & NS \\
Total cholesterol & $\mathbf{0 . 7 4 7}$ & $\mathbf{1 . 7 3 4}$ & NS \\
LDL-C & $\mathbf{0 . 7 1 2}$ & $\mathbf{1 . 1 9 3}$ & NS \\
HDL-C & $\mathbf{0 . 5 9 0}$ & $\mathbf{0 . 0 0 1}$ & HS \\
FT4 & $\mathbf{0 . 4 1 4}$ & $\mathbf{0 . 0 5}$ & S \\
FT3 & $\mathbf{0 . 2 3 1}$ & $\mathbf{0 . 0 1}$ & S \\
Anti-TPO & $\mathbf{0 . 2 9 8}$ & $\mathbf{0 . 7 8 2}$ & NS \\
Anti-TG & $\mathbf{0 . 9 3 5}$ & $\mathbf{0 . 0 0 3}$ & HS \\
Leptin & $\mathbf{0 . 3 6 0}$ & $\mathbf{0 . 0 3 8}$ & HS \\
Insulin & $\mathbf{0 . 2 8 7}$ & $\mathbf{0 . 0 0 2}$ & \\
IR & $\mathbf{0 . 4 5 3}$ & & \\
\hline
\end{tabular}

There were statistically significant correlation between abdominal ultrasonography results (hepatic steatosis) in the form of increased liver echogenecity with or without hepatomegaly with ALT, GGT, HbA1C, triglyceride, HDL-C, TSH, FT4, FT3, anti-TPO, anti-TG, serum insulin, insulin resistance (IR), and serum leptin but there were no statistically significant correlation between hepatic steatosis with AST, total bilirubin, prothrombin time, FBS, total cholesterol, and LDL-C as shown in table 6. 
Table 6: Correlation between the results of abdominal ultrasound (in the form of increased liver echogenicity alone, or both increased liver echogenicity and hepatomegaly) with laboratory parameters of our patients

\begin{tabular}{llll}
\hline Variables & Hepatic steatosis & \\
& $\mathbf{r}$ & P value & \\
ALT & $\mathbf{0 . 2 6 7}$ & $\mathbf{0 . 0 3 9}$ & $\mathbf{S}$ \\
AST & $\mathbf{0 . 7 2 0}$ & $\mathbf{1 . 0 4 5}$ & $\mathbf{N S}$ \\
GGT & $\mathbf{0 . 2 0 6}$ & $\mathbf{0 . 0 2 4}$ & $\mathbf{S}$ \\
Total bilirubin & $\mathbf{0 . 7 1 8}$ & $\mathbf{1 . 0 2 9}$ & $\mathbf{N S}$ \\
Prothrombin time & $\mathbf{0 . 4 8 6}$ & $\mathbf{0 . 0 8 7}$ & $\mathbf{N S}$ \\
FBS & $\mathbf{0 . 8 1 4}$ & $\mathbf{2 . 5 9 2}$ & $\mathbf{N S}$ \\
HbA1C & $\mathbf{0 . 2 7 2}$ & $\mathbf{0 . 0 3}$ & $\mathbf{S}$ \\
Triglyceride & $\mathbf{0 . 4 4 4}$ & $\mathbf{0 . 0 0 0 3}$ & HS \\
Total cholesterol & $\mathbf{0 . 8 3 0}$ & $\mathbf{2 . 3 7 1}$ & NS \\
LDL-C & $\mathbf{0 . 6 8 0}$ & $\mathbf{2 . 2 4 6}$ & NS \\
HDL-C & $\mathbf{0 . 4 1 5}$ & $\mathbf{0 . 0 0 1}$ & HS \\
TSH & $\mathbf{0 . 4 1 3}$ & $\mathbf{0 . 0 0 1}$ & HS \\
FT4 & $\mathbf{0 . 3 8 2}$ & $\mathbf{0 . 0 0 2}$ & HS \\
FT3 & $\mathbf{0 . 2 7 3}$ & $\mathbf{0 . 0 4}$ & $\mathbf{S}$ \\
Anti-TPO & $\mathbf{0 . 2 9 6}$ & $\mathbf{0 . 0 1}$ & $\mathbf{S}$ \\
Anti-TG & $\mathbf{0 . 3 4 5}$ & $\mathbf{0 . 0 0 2}$ & HS \\
IR & $\mathbf{0 . 3 8 0}$ & $\mathbf{0 . 0 0 2}$ & HS \\
Insulin & $\mathbf{0 . 2 7 0}$ & $\mathbf{0 . 0 2}$ & S \\
Leptin & $\mathbf{0 . 4 1 0}$ & $\mathbf{0 . 0 0 1}$ & HS \\
\hline
\end{tabular}

\section{Discussion}

Nonalcoholic fatty liver disease (NAFLD) defines a spectrum of histological abnormalities, from simple fatty liver to nonalcoholic steatohepatitis (NASH), in a person consuming no alcohol (9, 10,11). The main element of NAFLD is the accumulation of triglycerides (TG) as fat droplets within the cytoplasm of hepatocytes, which is a prerequisite for subsequent events of NASH, as more than $5 \%-10 \%$ of hepatocytes have fat droplets, as evident on liver biopsy (10). Increased delivery of both free fatty acids (FFA) and TG to the liver, diminished hepatic utilization of FFA, diminished export of TG from the liver, and impaired beta-oxidation of FFA within hepatocytes cause TG accumulation within the cytoplasm of hepatocytes $(12,13,14)$. Excess carbohydrate, either from dietary sources or de novo gluconeogenesis in the liver, is also a major stimulus for de novo fatty acid synthesis in the liver. Thus, fatty liver is caused by failure of normal hepatic fat metabolism either due to a defect within the hepatocyte or to delivary of excess fat, fatty acid and carbohydrate beyond the secretory capacity for lipids by the liver cells (15). Nonalcoholic fatty liver disease occurs equally in both genders (12). It is generally found within the fourth or fifth decade of life. Patients with NAFLD may have hypertension, besides obesity, type 2 diabetes, and hypertriglyceridemia ( $14,16,20)$. Many patients have no symptoms. The most frequent symptoms are right upper quadrant pain and dullness in a small number. It may present as diffuse smooth hepatomegaly such as obesity and diabetes mellitus $(16,17)$. The prevalence of NAFLD and NASH is $10 \%$ to $40 \%$ and about $2 \%$ to $5 \%$ in the American general adult population, respectively and $20 \%$ and $1.2 \%$ $4.8 \%$ in other developed countries. Hispanics may progress to cirrhosis more frequently than either blacks or whites $(18,19,20)$. Historically, an autopsy study showed a prevalence of NASH of $18.5 \%$ in markedly obese and $2.7 \%$ in lean subjects (20). Studies performed in general populations also showed that the prevalence of NASH is $3 \%$ in nonobese and $20 \%$ in obese subjects $(10,19,22)$. The prevalence of NASH related cirrhosis and hepatocellular carcinoma 
(HCC) is also high among patients with diabetes, as in obesity $(13,14,16)$. As a risk factor, hypertriglyceridemia is also associated with insulin resistance and NAFLD, even in patients without obesity $(11,23)$.

Hypothyroidism is a common endocrine disorder resulting from deficiency of thyroid hormone or, more rarely, from their impaired activity at tissue level. Prevalence is $1.9 \%$ in women, and it increases with age. . It may be a primary process in which the thyroid gland produces insufficient amounts of thyroid hormone (e.g. autoimmune thyroiditis, previous radio-iodine or surgical treatment of hyperthyroidism), but can also be secondary, that is, lack of thyroid hormone secretion due to inadequate secretion of either thyrotropin (that is, thyroid stimulating hormone [TSH]) from the pituitary gland or thyrotropinreleasing hormone (TRH) from the hypothalamus (secondary or tertiary hypothyroidism). The patient's presentation may vary from asymptomatic to, rarely, coma with multisystem organ failure (myxedema coma). The most common cause in the United States is autoimmune thyroid disease with antithyroid peroxidase antibodies (Hashimoto's thyroiditis). Individuals with subclinical hypothyroidism are often asymptomatic, but clinical manifestations can include non-specific complaints or symptoms similar to those seen in overt hypothyroidism, such as fatigue, weakness, weight gain, cold intolerance, and constipation (26). Hypothyroidism is characterized by a generalized reduction in metabolic function that most often manifests as a slowing of physical and mental activity with obesity. They may have diabetes and hypercholesterolemia and may present with weight gain, puffy face, hoarse and croaky voice, nonpitting edema in lower limbs, loss of outer third of eyebrow, dry skin, and bradycardia. There is also delayed relaxation of deep tendon reflexes, and hypertension $(24,25)$.

The findings from the present study showed a significant increased levels of thyroid stimulating hormone (TSH), anti-thyroid peroxidase ( antiTPO), and anti-thyroglobulin (anti-TG), but a significant low levels of both free thyroxin (FT4) and free tri-idothyronine (FT3) in our patients as compared with controls. Also, there was a higher prevalence of thyroid dysfunction in the form of overt and subclinical hypothyroidism among patients with NAFLD. The prevalence of overt and subclinical hypothyroidism in this study was reported to be $5 \%$ and $16.7 \%$ respectively among patients with NAFLD. As compared to healthy controls there was a significantly higher prevalence of hypothyroidism in patients with NAFLD compared to the controls. Our study was in agreement with a study done by Liangpunsakul and Chalasani in which the prevalence of hypothyroidism in patients with non-alcoholic steatohepatitis was $15 \%$ was significantly higher than in the controls $(7.2 \%, \mathrm{P}<0.001)$. By multivariate analysis, the prevalence of hypothyroidism in the non-alcoholic steatohepatitis group was significantly higher than in control group (27).

Several studies demonstrated that hypothyroidism is an independent risk factor for NAFLD. This indicates that hypothyroidism may directly result in NAFLD irrespective of other metabolic risk factors. Considering the results of these studies, hypothyroidism may be added to risk factors of NAFLD (28). The findings of our study were correlated with a study done by Chung et al who concluded that subclinical hypothyroidism, even in the range of upper normal TSH levels, was found to be related to NAFLD in a dosedependent manner. Hypothyroidism is closely associated with NAFLD independently of known metabolic risk factors, confirming a relevant clinical relationship between these two diseases (29).

Previous studies have investigated the association between NAFLD and thyroid function in adult or elderly patients $(29,30,32,33)$. The study by Targher et al. involving a large cohort of unselected adult outpatients was the first to report a strong association between thyroid function tests and serum liver enzyme activity concentrations (33). In particular, Targher et al. found a significant positive relationship between serum TSH, ALT, and GGT activities throughout the normal and high TSH ranges, and a similar inverse relationship between FT4 and serum liver enzyme activity concentrations (33). 
In our study, there was a significant positive association between hepatic steatosis (defined by the presence of a hyperechogenic ultrasound pattern of the liver and increased ALT concentrations) with TSH concentration, but inverse associations with FT4 and FT3 concentrations, thus both overt (5\%) and subclinical hypothyroidism (SH) $(16.7 \%)$ in our study was associated with hepatic steatosis. In another two studies, a significant inverse association between the FT4 concentration of NAFLD could be demonstrated, while no significant association could be identified for TT3 or TSH $(30,35)$. This underscores the importance of the TT4 or FT4 concentration as a marker for hepatic steatosis in the general population. By contrast, the TT3 concentration, in studies done by Ittermann et al. and $\mathrm{Xu}$ et al., had no identified value as a marker $(31,34)$. This finding could related to an inhibition of the conversion of TT4 to TT3, possibly explaining the subordinate diagnostic role of the TT3 or FT3. Even the study of Chung et al., which presented clear evidence of the association between hypothyroidism and NAFLD, did not ascribe any diagnostic value to the TT3 concentration (29). Beside the inverse association with FT4, Chung et al. and Xu et al. identified a positive association between NAFLD and TSH. This association was observed in other studies $(32,35)$.

In another study by Torun et al, he founded that elevated TSH level with normal FT3 and FT4 concentrations in obese subjects with NAFLD may occur as a result of resistance to thyroid hormones of peripheral tissues and a decreased negative feedback relationship between TSH and peripheral thyroid hormones (37). Subclinical hypothyroidism is also reported to be related to the relative low level of $\mathrm{T} 4$ binding protein in obese children (39). Chung etal stated that subclinical hypothyroidism has been found in NAFLD, and it has been reported that hypothyroidism is closely associated with NAFLD (29).

Subclinical hypothyroidism $(\mathrm{SCH})$ is defined as serum TSH level over $4.2 \mathrm{mIU} / \mathrm{L}$ with normal FT4 $(0.93$ - $1.71 \mathrm{ng} / \mathrm{ml})$ or FT3(2.57-4.43 pg/ml) concentrations. The most common cause of subclinical hypothyroidism is autoimmune thyroiditis especially Hashimoto's thyroiditis. Antibodies against TG and TPO are present in almost all patients with Hashimoto's thyroiditis (HT) (40). In addition to aiding the diagnosis, TPO antibodies can be used to help predict development of hypothyroidism, particularly when combined with measurement of TSH levels (41). In our study there was a positive correlation between TSH with anti-TPO antibodies but not correlated with anti-TG antibodies especially in patients with subclinical hypothyroidism, thus the most common cause of subclinical hypothyroidism in our patients may be related to Hashimoto's thyroiditis. Our study was in agreement with a study done by Assem et al. The results of this study revealed that a significant positive correlation between NAFLD and thyroid functions with higher prevalence of subclinical thyroid dysfunction in NAFLD patients. TPOantibodies in subclinical hypothyroid patients showed significant positive correlation with IR within the total study population. This study concluded that, in non-diabetic patients, IR and thyroid dysfunction have strong correlations with NAFLD and the role of thyroid autoimmunity in this relationship needs further assessment (42). Other studies fail to demonstrate any association between markers of thyroid dysfunction and presence of NAFLD. Despite lower free T3 levels among the NAFLD group, serum TSH, free T4, free T3, and markers of thyroid autoimmunity were not different in the participants with NAFLD and those without NAFLD. However, NAFLD patients were more likely to have low TSH levels although hypothyroidism and hyperthyroidism were not associated with NAFLD. The observed changes in TSH and free T3 levels may attribute to alterations in thyroid hormones due to sick euthyroid syndrome in $\operatorname{NAFLD}(28,43)$.

In our study, there was a significant increased levels of total cholesterol, LDL-C, triglycerides with significant low levels of HDL-C in our patients as compared with controls. Also, thyroid stimulating hormone (TSH) levels were significantly correlated with triglyceride levels but not correlated with total cholesterol, LDL-C, and HDL-C. Lastly, hepatic steatosis in our patients was significantly correlated with triglycerides and HDL-C levels. 
Our results correlated with a study by Musso et al, they stated that patients with NAFLD/NASH have abnormal lipid profiles notable for elevated cholesterol, low density lipoprotein and triglyceride levels (44). Thyroid hormones induce their effects on lipid metabolism via thyroid hormone receptor $\beta$, which is expressed in liver (45). Thyroid hormone receptor activation results in a reduction in body weight and fat as well as a decrease in cholesterol and triglyceride levels, which takes place only in hepatocytes $(46,47)$. Our study would confirm the correlation between NAFLD and thyroid dysfunction and a correlation between the TSH level and triglycerides (P $=0.003$ ). These observations agree with the findings of other studies that suggest a correlation between hypothyroidism and hyperlipidemia (48, 49). The increase in triglycerides in patients with hypothyroidism is explained by the reduced hepatic activity of triglyceride lipase $(48,50)$ and increased fatty acid oxidation. Loria et al. come to the conclusion that hepatic steatosis may develop from hypothyroidism induced hyperlipidemia and overweight (51). The development of hepatic steatosis may be explained by an increase influx of triglycerides and an imbalance between the inand outflow of other lipids in the liver.

The findings from our study revealed that, there were significant increased levels of FBS, HbA1C, serum insulin, insulin resistance (IR), and serum leptin in our patients as compared with controls. Plasma levels of TSH were correlated significantly with levels of HbA1C, leptin, insulin and insulin resistance. Also, hepatic steatosis was significantly correlated with $\mathrm{HbA} 1 \mathrm{C}$, serum insulin, insulin resistance and serum leptin levels in our patients.

Our results were in agreement with a study done by Roos et al, and Park et al that identified a significant association between thyroid dysfunction, a hypothyroid metabolic state and the metabolic syndrome $(48,50)$. Metabolic syndrome, in turn, is associated with NAFLD (44, $52,53)$ and would point indirectly to a possible correlation between thyroid dysfunction and NAFLD. Non-alcoholic fatty liver disease (NAFLD) is supposed to be a hepatic feature of metabolic syndrome and insulin resistance (54,
55). Hypothyroidism has been reported to be associated with obesity and metabolic syndrome $(55,56,57)$. Insulin resistance in the context of hypothyroidism and its alleviation with treatment of the hypothyroidism has been reported (58).

Hypothyroidism was more prevalent in patients with type 2 diabetes and in some reports was associated with diabetic microangiopathy (59). Associations of hypothyroidism with these metabolic abnormalities, which are frequently accompanied by NAFLD, strengthen the notion of association between hypothyroidism and NAFLD. While the underlying pathophysiology for this association is still not clear, several mechanisms have been proposed. The role of adipocytokines in NAFLD $(60,61)$ has been established previously, and some studies aimed to find a relationship between adipocytokines and hypothyroidism to clarify the mechanism of thyroid dysfunction and NAFLD. An increased level of leptin has been identified in patients with hypothyroidism, and it may be responsible for the development of NAFLD/NASH in this context $(62,63)$. Leptin is an adipocytokine involved in the regulation of appetite, with an increased level seen in cases of obesity, can induce collagen synthesis in the liver and promotes hepatic insulin resistance $(64,65,66)$. Our study was correlated with the study by Aeberli et al. who prospectively examined the associations between changes in thyroid function, IR, and other metabolic risk factors in obese children undergoing weight and fat loss in a well-controlled 8-week inpatient program, changes in TSH did not correlate with losses of weight, fat, or lean tissue (as assessed by dual energy X-ray absorption), but they significantly correlated with fasting insulin and HOMA-IR, independently of body weight and body composition (67). In our study, IR increased as the degree of steatosis increased parallel to increased TSH levels and there was a relationship between TSH and IR in correlation analysis. The results of our study were correlated with the results of Pacifico et al, they stated that IR, leading to impaired hepatic glucose production and glucose uptake in muscle is a component of the MS (68). Recent studies have revealed that subclinical hypothyroidism worsens IR (69). It has been reported that increasing levels of TSH 
and decreasing levels of FT4 are associated with increased IR (68).

The present study has some limitations, first it is a short prospective design which permitted an examination of association, not causation. The diagnosis of NAFLD in our study was based on ultrasound data and the severity of liver disease was not confirmed histologically.

\section{Conclusion}

The results of the present study confirm an association between increased TSH concentrations and hepatic steatosis. The prevalence of hepatic steatosis rises significantly with elevations in TSH concentrations. An increased TSH concentration appears to be an independent risk factor for hepatic steatosis. There were significant correlations between TSH with fasting insulin, HOMA-IR and Leptin. We found that elevated TSH is a significant predictor of lipid and glucose dysmetabolism, hepatic insulin resistance as well as of hepatic steatosis, independently of the degree of total and central obesity. Nonetheless, the possibility that NAFLD and thyroid hormones share common genetic, autoimmune or environmental influences accounting for the observed association cannot be discounted. However, from this study thyroid hormone profiles may be tested as a part of initial clinical assessment in patients with NAFLD and if treatment of hypothyroidism in patients with NAFLD with thyroid replacement therapy will improve disease progression and outcome or not. Future studies should further elucidate the impact of NAFLD on thyroid hormone parameters. This could serve to clarify the underlying pathogenetic mechanisms and provide potentially important data for diagnostic, therapeutic and preventive measures.

\section{References}

1. Falck-Ytter Y, Younossi ZM, Marchesini G, McCullough AJ. Clinical features and natural history of nonalcoholic steatosis syndromes. Semin Liver Dis 2001;21:1726.
2. Angulo P. GI epidemiology: nonalcoholic fatty liver disease. Aliment Pharmacol Ther 2007;25:883-889.

3. Yamada T, Fukatsu M, Suzuki S, Wada T, Yoshida T, Joh T. Fatty liver predicts impaired fasting glucose and type 2 diabetes mellitus in Japanese undergoing a health checkup. J Gastroenterol Hepatol 2010;25:352-356.

4. Caldwell SH, Lee VD, Kleiner DE, AlOsaimi AM, Argo CK, Northup PG, et al. NASH and cryptogenic cirrhosis: a histological analysis. Ann Hepatol 2009;8:346-352.

5. Matteoni CA, Younossi ZM, Gramlich T, Boparai N, Liu YC, McCullough AJ. Nonalcoholic fatty liver disease: a spectrum of clinical and pathological severity. Gastroenterology 1999;116:1413-1419.

6. Rodondi N, den Elzen WP, Bauer DC, Cappola AR, Razvi S, Walsh JP, et al. Subclinical hypothyroidism and the risk of coronary heart disease and mortality. JAMA 2010;304:1365-1374.

7. Alam MJ, Rahman S, Al-Matab $M$. Association of fatty liver and hypothyroidism.Euroasian J Hepatogastroenterol 2013, 3(1): 8-9.

8. Angel I (1988) The use of microtiter plates for the simple and sensitive determination of insulin by an ELISA method. Experientia 44: 877-879.

9. Bril F, Lomonaco R, Orsak B, et al. Relationship between disease severity, hyperinsulinemia, and impaired insulin clearance in patients with nonalcoholic steatohepatitis. Hepatology 2014; 59: 2178-87.

10. Tilg H, Moschen AR. Evolving therapies for non-alcoholic steatohepatitis. Expert Opin Drug Discov 2014; 9: 687-96.

11. Miyake T, Kumagi T, Hirooka M, et al. Significance of exercise in nonalcoholic fatty liver disease in men: a communitybased large cross-sectional study. J Gastroenterol 2014. 
12. Neuschwander-Tetri BA, Caldwell SH. Nonalcoholic steatohepatitis: summary of an AASLD Single Topic Conference. Hepatology 2003; 37: 1202-19.

13. Browning JD, Horton JD. Molecular mediators of hepatic steatosis and liver injury. J Clin Invest 2004; 114: 147-52.

14. Crespo J, Cayón A, Fernández-Gil P, et al. Gene expression of tumor necrosis factor a and TNF-receptors, p55 and p75, in nonalcoholic steatohepatitis patients. Hepatology 2001; 34: 1158-63.

15. Bacon BR, Faravash MJ, Janny CG, et al. Non-alcoholic steatohepatitis: An expanded clinical entity. Gastroenterology 1994; 107:1103.

16. Yang SQ, Lin HZ, Lane MD, Clemens M, Diehl AM. Obesity increases sensitivity to endotoxin liver injury: implications for the pathogenesis of steatohepatitis. Proc Natl Acad Sci U S A 1997; 94: 2557-62.

17. Zhang X, Harmsen WS, Mettler TA, et al. Continuation of metformin use after a diagnosis of cirrhosis significantly improved survival of patients with diabetes. Hepatology 2014. doi: 10.1002/ hep. 27199.

18. Sonsuz A, Basaranoglu M, Bilir M, Senturk H, Akin P. Hyperinsulinemia in nondiabetic, both obese and nonobese patients with nonalcoholic steatohepatitis. Am J Gastroenterol 2002; 97: 495.

19. Caldwell SH, Harris DM, Patrie JT, Hespenheide EE. Is NASH underdiagnosed among African Americans? Am J Gastro 2002; 97: 1496500.

20. Browning JD, Kumar KS, Saboorian MH, Thiele DL. Ethnic differences in the prevalence of cryptogenic cirrhosis. Am J Gastro 2004; 99: 292-8.

21. Wanless IR, Lentz JS. Fatty liver hepatitis (steatohepatitis) and obesity: an autopsy study with analysis of risk factors. Hepatology 1990; 12: 1106-10.

22. Basaranoglu M, Sonsuz A, Senturk H, Akin P. The low incidence of primary liver disease in patients with nonalcoholic steatohepatitis. J Hepatol 2001; 35: 684-5.
23. Tilg H, Moschen AR. Evolving therapies for non-alcoholic steatohepatitis. Expert Opin Drug Discov 2014; 9: 687-96.

24. Bello F and Bakari A. G. Hypothyroidism in adults: A review and recent advances in management. Journal of Diabetes and Endocrinology 2012;3(5):57-69.

25. Nicoloff JT, LoPresti JS (2007). Hypothyroidism. In: Rakel RE, Bope ET, (eds), Conn's Current Therapy. Saunders Elsevier, Philadelphia. pp. 766-771.

26. Biondi B, Cooper DS. The clinical significance of subclinical thyroid dysfunction. Endocr Rev. 2008;29(1):76131.

27. Liangpunsakul S, Chalasani N. Is hypothyroidism a risk factor for nonalcoholic steatohepatitis? J Clin Gastroenterol. 2003;37:340-3.

28. Eshraghian A, Hamidian Jahromi A. Nonalcoholic fatty liver disease and thyroid dysfunction: A systematic review. World $\mathbf{J}$ Gastroenterol 2014; 20(25): 8102-8109.

29. G. E. Chung, D. Kim,W. Kim et al., "Nonalcoholic fatty liver disease across the spectrum of hypothyroidism," Journal of Hepatology, vol. 57, no. 1, pp. 150-156, 2012.

30. M. R. Pagadala, C. O. Zein, S. Dasarathy, L. M. Yerian, R. Lopez, and A. J.McCullough, "Prevalence of hypothyroidism in nonalcoholic fatty liver disease," Digestive Diseases and Sciences, vol. 57, no. 2, pp. 528-534, 2012.

31. T. Ittermann, R.Haring,H.Wallaschofski et al., "Inverse association between serumfree thyroxine levels and hepatic steatosis: results from the study of health in Pomerania," Thyroid, vol. 22, no. 6, pp. 568-574, 2012.

32. L. Carulli, S. Ballestri, A. Lonardo et al., "Is nonalcoholic steatohepatitis associated with a high-though-normal thyroid stimulating hormone level and lower cholesterol levels?" Internal and Emergency Medicine. Intern Emerg Med 2013; 8: 297-305. 
33. G. Targher, M. Montagnana, G. Salvagno et al., "Association between serum TSH, free T4 and serum liver enzyme activities in a large cohort of unselected outpatients," Clinical Endocrinology, vol. 68, no. 3, pp. 481-484, 2008.

34. $\mathrm{Xu} \mathrm{C,} \mathrm{Xu} \mathrm{L,} \mathrm{Yu} \mathrm{C,} \mathrm{Miao} \mathrm{M,} \mathrm{Li} \mathrm{Y.}$ Association between thyroid function and nonalcoholic fatty liver disease in euthyroid elderly Chinese. Clin Endocrinol (Oxf). 2011;75:240-6.

35. Moustafa AH, Ali EM, Mohamed TM, Abdou HI. Oxidative stress and thyroid hormones in patients with liver diseases. Eur J Intern Med. 2009;20:703-8.

36. Ludwig U, D Holzner D, Denzer C, Greinert A1, Haenle MM, Oeztuerk S, Koenig W, Boehm BO, Mason RA, Kratzer W, Graeter $\mathrm{T}$ and the EMILStudy. Subclinical and clinical hypothyroidism and non-alcoholic fatty liver disease: a cross-sectional study of a random population sample aged 18 to 65 years. BMC Endocrine Disorders (2015) $15: 41$.

37. Torun E, Ozgen IT, Gokce S, Aydın S, Cesur Y. Thyroid Hormone Levels in Obese Children and Adolescents with Non-Alcoholic Fatty Liver Disease. J Clin Res Pediatr Endocrinol 2014;6(1):34-39

38. Reinehr $\mathrm{T}$ andler W. Thyroid hormones before and after weight loss in obesity. Arc Dis Child 2002;87:320-323.

39. Uzunlulu M, Yorulmaz E, Oguz A. Prevalence of subclinical Hypothyroidism in Patients with metabolic syndrome. Endocr J 2007;54:71-76. Epub 2006 Nov 14

40. Weetman AP. Cellular immune responses in autoimmune thyroid disease. Clin Endocrinol (Oxf) 2004; 61: 405-413.

41. Walsh JP, Bremner AP, Feddema P, Leedman PJ, Brown SJ, O'Leary P. Thyrotropin and thyroid antibodies as predictors of hypothyroidism: a 13-year, longitudinal study of a community-based cohort using current immunoassay techniques. J Clin Endocrinol Metab 2010; 95: 1095-1104.
42. Assem M, Fawzi M, Ibrahim A \& Saif A. Assessment of thyroid functions, thyroid autoimmunity and insulin resistance in non-diabetic patients with non-alcoholic fatty liver disease. Endocrine Abstracts (2017) 49 EP1240.

43. Lee KW, Bang KB, Rhee EJ, Kwon HJ, Lee MY, and Cho YK. Impact of hypothyroidism on the development of non-alcoholic fatty liver disease: A 4-year retrospective cohort study. Clinical and Molecular Hepatology 2015;21:372-378.

44. Musso G, Gambino R, Cassader M. Recent insights into hepatic lipid metabolism in non-alcoholic fatty liver disease (NAFLD). Prog Lipid Res 2009; 48: 1-26.

45. Hulbert AJ. Thyroid hormones and their effects: a new perspective. Biol Rev Camb Philos Soc 2000; 75: 519-631.

46. Grover GJ, Mellström K, Ye L, Malm J, Li YL, Bladh LG, Sleph PG, Smith MA, George R, Vennström B, Mookhtiar K, Horvath R, Speelman J, Egan D, Baxter JD. Selective thyroid hormone receptorbeta activation: a strategy for reduction of weight, cholesterol, and lipoprotein (a) with reduced cardiovascular liability. Proc Natl Acad Sci USA 2003; 100: 1006710072.

47. Erion MD, Cable EE, Ito BR, Jiang $\mathrm{H}$, Fujitaki JM, Finn PD, Zhang BH, Hou J, Boyer SH, van Poelje PD, Linemeyer DL. Targeting thyroid hormone receptor-beta agonists to the liver reduces cholesterol and triglycerides and improves the therapeutic index. Proc Natl Acad Sci USA 2007; 104: 15490-15495.

48. Roos A, Bakker SJ, Links TP, Gans RO, Wolffenbuttel BH. Thyroid function is associated with components of the metabolic syndrome in euthyroid subjects. J Clin Endocrinol Metab. 2007;92:491-6.

49. Tagami T, Kimura H, Ohtani S, Tanaka T, Tanaka T, Hata S, et al. PHPH study group: multi-center study on the prevalence of hypothyroidism in patients with hypercholesterolemia. Endocr J. 2011;58:449-57. 
50. Park HT, Cho GJ, Ahn KH, Shin JH, Hong SC, Kim $\mathrm{T}$, et al. Thyroid stimulating hormone is associated with metabolic syndrome in euthyroid postmenopausal women. Maturitas. 2009;62:301-5.

51. Loria P, Carulli L, Bertolotti M, Lonardo A. Endocrine and liver interaction: the role of endocrine pathways in NASH. Nat Rev Gastroenterol Hepatol. 2009;6:236-47.

52. Zelber-Sagi S, Nitzan-Kaluski D, Halpern Z, Oren R. Prevalence of primary nonalcoholic fatty liver disease in a population-based study and its association with biochemical and anthropometric measures. Liver Int. 2006;26:856-63.

53. Bookman ID, Pham J, Guindi M, Heathcote EJ. Distinguishing nonalcoholic steatohepatitis from fatty liver: serum-free fatty acids, insulin resistance, and serum lipoproteins. Liver Int. 2006;26:566-71.

54. Collantes RS, Ong JP, Younossi ZM. The metabolic syndrome and nonalcoholic fatty liver disease. Panminerva Med 2006; 48: 41-48

55. Pearce EN. Thyroid hormone and obesity. Curr Opin Endocrinol Diabetes Obes 2012; 19: 408-413

56. Pacifico L, Anania C, Ferraro F, Andreoli GM, Chiesa C. Thyroid function in childhood obesity and metabolic comorbidity. Clin Chim Acta 2012; 413: 396-405.

57. Waring AC, Rodondi N, Harrison S, Kanaya AM, Simonsick EM, Miljkovic I, Satterfield S, Newman AB, Bauer DC. Thyroid function and prevalent and incident metabolic syndrome in older adults: the Health, Ageing and Body Composition Study. Clin Endocrinol (Oxf) 2012; 76: 911-918.

58. Kowalska I, Borawski J, Nikołajuk A, Budlewski T, Otziomek E, Górska M, Strączkowski M. Insulin sensitivity, plasma adiponectin and sICAM-1 concentrations in patients with subclinical hypothyroidism: response to levothyroxine therapy. Endocrine 2011; 40: 95-101
59. Díez JJ, Iglesias P. Subclinical hyperthyroidism in patients with type 2 diabetes. Endocrine 2012; 42: 157-163.

60. Jarrar MH, Baranova A, Collantes R, Ranard B, Stepanova M, Bennett C, Fang Y, Elariny H, Goodman Z, Chandhoke V, Younossi ZM. Adipokines and cytokines in non-alcoholic fatty liver disease. Aliment Pharmacol Ther 2008; 27: 412421.

61. Musso G, Gambino R, Durazzo M, Biroli G, Carello M, Fagà E, Pacini G, De Michieli F, Rabbione L, Premoli A, Cassader M, Pagano G. Adipokines in NASH: postprandial lipid metabolism as a link between adiponectin and liver disease. Hepatology 2005; 42: 1175-1183.

62. Leonhardt U, Ritzel U, Schäfer G, Becker W, Ramadori G. Serum leptin levels in hypo- and hyperthyroidism. $J$ Endocrinol 1998; 157: 75-79.

63. Kautzky-Willer A, Ludwig C, Nowotny P, Roden A, Huemer C, Widhalm K, Vierhapper H, Waldhäusl W, Roden M. Elevation of plasma leptin concentrations in obese hyperinsulinaemic hypothyroidism before and after treatment. Eur J Clin Invest 1999; 29: 395-403.

64. Oswal A, Yeo G. Leptin and the control of body weight: a review of its diverse central targets, signaling mechanisms, and role in the pathogenesis of obesity. Obesity (Silver Spring) 2010; 18: 221-229.

65. Saxena NK, Ikeda K, Rockey DC, Friedman SL, Anania FA. Leptin in hepatic fibrosis: evidence for increased collagen production in stellate cells and lean littermates of ob/ob mice. Hepatology 2002; 35: 762-771.

66. Benomar Y, Wetzler S, Larue-Achagiotis $\mathrm{C}$, Djiane J, Tomé $\mathrm{D}$, Taouis $\mathrm{M}$. In vivo leptin infusion impairs insulin and leptin signalling in liver and hypothalamus. Mol Cell Endocrinol 2005; 242: 59-66. 
67. I. Aeberli, A. Jung, S. B. Murer et al., "During rapid weight loss in obese children, reductions in TSH predict improvements in insulin sensitivity independent of changes in body weight or fat," Journal of Clinical Endocrinology and Metabolism, vol. 95, no. 12, pp. 5412-5418, 2010.

68. Pacifico L, Anania C, Ferraro F andreoli GM, Chiesa C. Thyroid function in childhood obesity and metabolic comorbidity. Clin Chim Acta 2012;413:396-405. Epub 2011 Nov 27

69. Maratou E, Hadjidakis DJ, Kollias A, Tsegka K, Peppa M, Alevizaki M, Mitrou P, Lambadiari V, Boutati E, Nikzas D, Tountas N,Economopoulos T, Raptis SA, Dimitriadis G. Studies of insulin resistance in patients with clinical and subclinical hypothyroidism. Eur J Endocrinol 2009;160:785-790. Epub 2009 Jan 13

\begin{tabular}{|c|c|}
\hline \multicolumn{2}{|c|}{ Access this Article in Online } \\
\hline \multirow{2}{*}{ 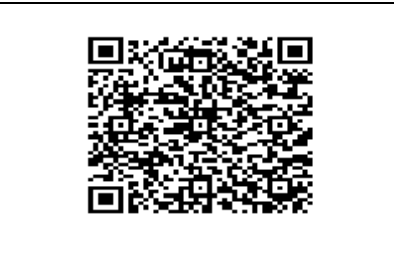 } & $\begin{array}{l}\text { Website: } \\
\text { www.ijcrims.com }\end{array}$ \\
\hline & $\begin{array}{l}\text { Subject: } \\
\text { Medicine }\end{array}$ \\
\hline Quick Response Code & \\
\hline
\end{tabular}

How to cite this article:

Arafat Kassem, Farag Khalil, Mokhtar Ragab Ramadan, Mohamed Rashed. (2017). Association and impact of non-alcoholic fatty liver disease on thyroid function. Int. J. Curr. Res. Med. Sci. 3(7): 94-107.

DOI: http://dx.doi.org/10.22192/ijcrms.2017.03.07.016 